\title{
TRANSFORMER IN ACTION: A COMPARATIVE STUDY OF TRANSFORMER-BASED ACOUSTIC MODELS FOR LARGE SCALE SPEECH RECOGNITION APPLICATIONS
}

\author{
Yongqiang Wang*, Yangyang Shi*, Frank Zhang*, Chunyang $\mathrm{Wu}^{*}$, \\ Julian Chan*, Ching-Feng Yeh, Alex Xiao
}

Facebook AI, USA

\begin{abstract}
In this paper, we summarize the application of transformer and its streamable variant, Emformer based acoustic model for large scale speech recognition applications. We compare the transformer based acoustic models with their LSTM counterparts on industrial scale tasks. Specifically, we compare Emformer with latency-controlled BLSTM (LCBLSTM) on medium latency tasks and LSTM on low latency tasks. On a low latency voice assistant task, Emformer gets $24 \%$ to $26 \%$ relative word error rate reductions (WERRs). For medium latency scenarios, comparing with LCBLSTM with similar model size and latency, Emformer gets significant WERR across four languages in video captioning datasets with 2-3 times inference real-time factors reduction.
\end{abstract}

Index Terms - speech recognition, acoustic modeling, transformer, recurrent neural networks

\section{Introduction}

Since the introduction of deep learning into automatic speech recognition (ASR) [1], architectures of the neural acoustic models have evolved from feed-forward networks (e.g., [2]) to recurrent neural networks (e.g. long short term memory network, LSTM [3]), convolution neural networks (e.g. [4]) and their variants, e.g. latencyconstrained bidirectional LSTM (LCBLSTM) [5], time-delay neural networks (TDNN) [6], feed-forward sequential memory networks (FSMN) [7] to name a few. Recently, inspired by the great success achieved by transformer networks $[8]$ in natural language processing [9] and machine translation, self-attention based transformer acoustic models have demonstrated promising results in terms of both modeling accuracy and computational efficiency [10 14]. Understandably, to prove the concept, these works focused on improving the absolute word error rates (WERs) on relatively small to mediumsized public datasets, with no production constraints, such as latency and real-time factors (RTFs). This work aims to close the gap by presenting a comprehensive comparative study of transformer-based and the widely used LSTM and its variant LCBLSTM-based acoustic models on several of our internal industrial scale datasets, whose training data ranges from 9,000 (supervised) hours to 2.2 million (semi-supervised) hours.

There is a board spectrum of speech applications powered by ASR technology, resulting in very different requirements for ASR systems. For example, audio indexing and caption generation for video on-demand impose little to no latency constraints, but the system must be able to process a large amount of incoming traffic at a high throughput; on the other hand, users are usually sensitive to the response latency when interacting with a voice interface. As the first step in the response generation pipeline, a low latency induced by ASR systems is critical. Caption generation for live video sits be-

\footnotetext{
$\star$ Equal contribution.
}

tween these two extremes, where a medium latency (e.g., up to 1s) is acceptable. Different neural acoustic models have been invented to fit these applications. For example, LSTM-based neural encoders are widely used for voice interface applications due to its very small algorithmic latency; LCBLSTM-based acoustic models are widely used for medium latency applications, while BLSTM based acoustic model can be used for full-offline batch processing.

In [11], we have demonstrated that the transformer-based acoustic model can significantly outperform the BLSTM-based model in terms of both modeling accuracy and inference speed. However, it is not able to process streaming audio. In [15], an augmented memory transformer (AM-TRF) model is proposed to make the transformer streamable. On top of AM-TRF, [16] proposed an efficient memory transformer, Emformer, which further improves both training and inference speed. In this work, we will demonstrate that Emformer achieves significantly better recognition accuracies and faster RTFs on several large scale speech recognition tasks, covering most of the low-to-medium latency scenarios. Our comparison is rigorously performed under practical production settings. We hope that by presenting these results, we can convince readers that our proposed Emformer-based acoustic models can be a competitive solution for either traditional hybrid or end-to-end style neural transducer [17] ASR systems.

\section{Acoustic Models}

In both hybrid [18] and neural transducer architecture, an acoustic mode 1 is an important component. It is used to encode an input sequence $\boldsymbol{x}_{1}, \cdots, \boldsymbol{x}_{T}$ to a sequence of high level acoustic embedding vectors $\boldsymbol{z}_{1}, \cdots, \boldsymbol{z}_{T}$. In the hybrid architecture, these embedding vectors are used to produce a posterior distribution of tied states of hidden Markov models (HMMs), such as senone [19] or chenone [20]. Cross entropy or connectionist temporal classification (CTC) $|21|$ criterion can be used as the loss function. This is usually followed by a state-level minimum Bayes risk (sMBR) training [22]. In the neural transducer architecture, these acoustic embeddings are combined with the embeddings from transcription networks via a joiner network to produce a posterior distribution over the modeling units, usually characters or wordpieces [23]. A beam search is then performed to find the best hypothesis.

\subsection{LSTM-based acoustic models}

In practise, unidirectional LSTM-based acoustic models are widely used in low latency ASR scenarios. Given an input sequence to the $n$-th LSTM layer, $\boldsymbol{x}_{0}^{n}, \cdots, \boldsymbol{x}_{T-1}^{n}$, its output (or input to the next layer) is calculated by:

$$
\boldsymbol{x}_{0}^{n+1}, \cdots, \boldsymbol{x}_{T-1}^{n+1}=\operatorname{LSTM}\left(\boldsymbol{x}_{0}^{n}, \cdots, \boldsymbol{x}_{T-1}^{n} ; \boldsymbol{c}_{0}^{n}, \boldsymbol{h}_{0}^{n}\right)
$$

\footnotetext{
${ }^{1}$ It is also referred to as encoder in the end-to-end modelling. We will use acoustic model and encoder interchangeably.
} 
where $\operatorname{LSTM}(\cdot)$ is the function to denote LSTM cell operations on the input sequence; $\boldsymbol{c}_{0}^{n}$ and $\boldsymbol{h}_{0}^{n}$ are the initial cell and hidden state. Note that in Eq. 11, $\boldsymbol{x}_{t}^{n+1}$ is a function of $\boldsymbol{x}_{0}^{n}$ until $\boldsymbol{x}_{t}^{n}$, thus introduce no algorithmic latency. In the application scenarios which allows medium latency, latency controlled bi-directional LSTM is often used. The input sequence is first chunked into a sequence of non-overlapping segments, $\mathbf{C}_{0}^{0}, \cdots, \mathbf{C}_{k}^{0}, \cdots, \mathbf{C}_{K-1}^{0}$, where $\mathbf{C}_{k}^{0} \in \mathbb{R}^{c \times d}, c$ is the number of frames in this segment, $d$ is the dimension of individual vectors, the subscript $k$ means the index of the segments, and superscript 0 here means that these are the input the 0 -th layer. To allow the acoustic model to look ahead, a right context block $\mathbf{R}_{k}^{0} \in \mathbb{R}^{r \times d}$ with $r$ look-ahead frames is concatenated with $\mathbf{C}_{k}^{0}$. For the $k$-th segment, the $n$-th layer processes its input $\left[\mathbf{C}_{k}^{n}, \mathbf{R}_{k}^{n}\right]$ in the following way:

$$
\begin{gathered}
{\left[\overrightarrow{\mathbf{C}}_{k}^{n+1}, \overrightarrow{\mathbf{R}}_{k}^{n+1}\right]=\operatorname{LSTM}_{1}\left(\left[\mathbf{C}_{k}^{n}, \mathbf{R}_{k}^{n}\right] ; \mathbf{h}_{k-1}^{n}, \mathbf{c}_{k-1}^{n}\right)} \\
{\left[\overleftarrow{\mathbf{C}}_{k}^{n+1}, \overleftarrow{\mathbf{R}}_{k}^{n+1}\right]=\mathcal{R}\left(\operatorname{LSTM}_{\mathrm{r}}\left(\mathcal{R}\left(\left[\mathbf{C}_{k 1}^{n}, \mathbf{R}_{k}^{n}\right]\right) ; \mathbf{0}, \mathbf{0}\right)\right)} \\
\mathbf{C}_{k}^{n+1}=\left[\overrightarrow{\mathbf{C}}_{k}^{n+1}, \overleftarrow{\mathbf{C}}_{k}^{n+1}\right]^{\top} \quad \mathbf{R}_{k}^{n+1}=\left[\overrightarrow{\mathbf{R}}_{k}^{n+1}, \overleftarrow{\mathbf{R}}_{k}^{n+1}\right]^{\top}
\end{gathered}
$$

where $\mathrm{LSTM}_{1}$ and $\mathrm{LSTM}_{\mathrm{r}}$ is the left-to-right LSTM and rightto-left LSTM respectively. $\mathcal{R}(\cdot)$ is to reverse the order of a sequence; and $\mathbf{h}_{k-1}^{n}$ and $\mathbf{c}_{k-1}^{n}$ are the $n$-th layer's hidden and cell state at the last frame of $(k-1)$-th segment. At the last layer, $\mathbf{C}_{0}^{N-1}, \cdots, \mathbf{C}_{K-1}^{N-1}$ are assembled to form the sequence of acoustic embeddings.

\subsection{Transformer-based acoustic models}

For a particular transformer layer, there are two components: the self attention module and the feed-forward network (FFN) module. Given the input sequence to the $n$-th transformer layer, $\mathbf{X}^{n}=$ $\left[\boldsymbol{x}_{0}^{n}, \cdots, \boldsymbol{x}_{T-1}^{n}\right]$, the self attention module performs the following operations:

$$
\begin{aligned}
& \hat{\mathbf{X}}^{n}=\operatorname{LayerNorm}\left(\mathbf{X}^{n}\right) \\
& \mathbf{Q}^{n}=\mathbf{W}_{\mathrm{q}} \hat{\mathbf{X}}^{n}, \mathbf{K}^{n}=\mathbf{W}_{\mathrm{k}} \hat{\mathbf{X}}^{n}, \mathbf{V}^{n}=\mathbf{W}_{\mathbf{v}} \hat{\mathbf{X}}^{n} \\
& \mathbf{Z}^{n}=\operatorname{Attn}\left(\mathbf{Q}^{n}, \mathbf{K}^{n}, \mathbf{V}^{n}\right)+\mathbf{X}^{n}
\end{aligned}
$$

where LayerNorm is the layer normalization operation [24], $\mathbf{W}_{\mathrm{q}}, \mathbf{W}_{\mathrm{k}}$ and $\mathbf{W}_{\mathrm{v}}$ are the projection matrices for query, key and value, respectively. $\operatorname{Attn}(\cdot)$ is the attention mechanism first proposed in [25]. Note that Eq. (7) uses a residual connection. After this attention module, $\mathbf{Z}^{n}$ is fed to the FFN module equipped with layer normalization and residual connection; usually a two-layer linear transformer with Relu non-linearity is used, i.e.,

$$
\begin{aligned}
\hat{\mathbf{Z}}^{n} & =\operatorname{FFN}\left(\text { LayerNorm }\left(\mathbf{Z}^{n}\right)\right)+\mathbf{Z}^{n} \\
\mathbf{X}^{n+1} & =\operatorname{LayerNorm}\left(\hat{\mathbf{Z}}^{n}\right)
\end{aligned}
$$

Note that the last layer normalization in Eq. 97 is needed to prevent a path which can bypass all the transformer layers.

The attention operation in Eq. (7) requires access to every element in the input sequence, which makes it impossible for streaming inputs. It also incurs a quadratically growing computational cost with respect to the input sequence. To make it possible to process audio signal incrementally, [15] proposed the augmented memory transformer (AM-TRF). Similar to LCBLSTM, AM-TRF uses block processing to deal with incrementally arrived sequences: a fixed size left and right context block, $\mathbf{L}_{k}^{n} \in \mathbb{R}^{l \times d}$ and $\mathbf{R}_{k}^{n} \in \mathbb{R}^{r \times d}$ are concatenated with the $k$-th segmeg $\mathbf{C}_{k}^{n} \in \mathbb{R}^{c \times d}$ to form a contextual segment $\mathbf{X}_{k}^{n}=\left[\mathbf{L}_{k}^{n}, \mathbf{C}_{k}^{n}, \mathbf{R}_{k}^{n}\right]$. At the $k$-th segment, the $n$-th AM-TRF layer accepts $\mathbf{X}_{k}^{n}$ and a bank of memory vectors $\mathbf{M}_{k}^{n}=$ $\left[\mathbf{m}_{0}^{n}, \cdots, \mathbf{m}_{k-1}^{n}\right]$ as the input, and produces $\mathbf{X}_{k}^{n+1}$ and $\mathbf{m}_{k}^{n}$ as the output, whereas $\mathbf{X}_{k}^{n+1}$ is fed to the next layer and $\boldsymbol{m}_{k}^{n}$ is inserted into the memory bank to generate $\mathbf{M}_{k+1}^{n}$ and carried over to the next segment. After all the AM-TRF layers, the center blocks $\left\{\mathbf{C}_{k}^{N-1}\right\}_{k=0}^{K-1}$ are concatenated as the encoder output sequence.

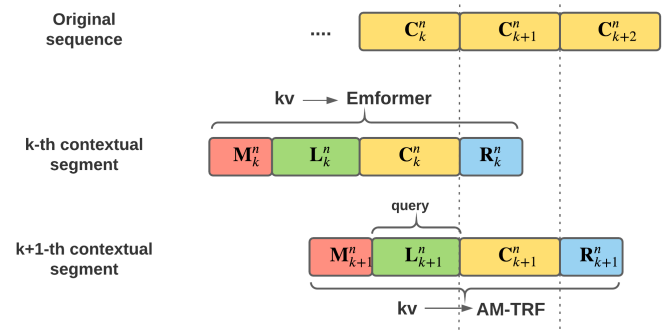

Fig. 1: Comparison of AM-TRF with Emformer on computing embedding for $\mathbf{L}_{k+1}^{n}$. Emformer allows attention to previous contextual segment for query $\mathbf{L}_{k+1}^{n}$, therefore enables caching the embedding for left context blocks.

One limitation of AM-TRF is that the embedding for the left context block $\mathbf{L}_{k}^{n}$ needs to be re-computed for every step, even though $\mathbf{L}_{k}^{n}$ is overlapped with $\mathbf{C}_{k-1}^{n}$ (or possibly even more previous center blocks). This is illustrated in Figure 1 In [16], we proposed an efficient memory transformer, Emformer, in which when compute the attention output for $\mathbf{L}_{k+1}^{n}$, the attention attend to $\left[\mathbf{X}_{k}^{n}, \mathbf{m}_{k}^{n}\right]$, which has been computed already in the past, therefore we can save the computation for the left context blocks, which improves both the training and inference efficiency. The forward pass procedure in Emformer is illustrated in Algorithm 1 More detail of Emformer can be found in our companion paper [16].

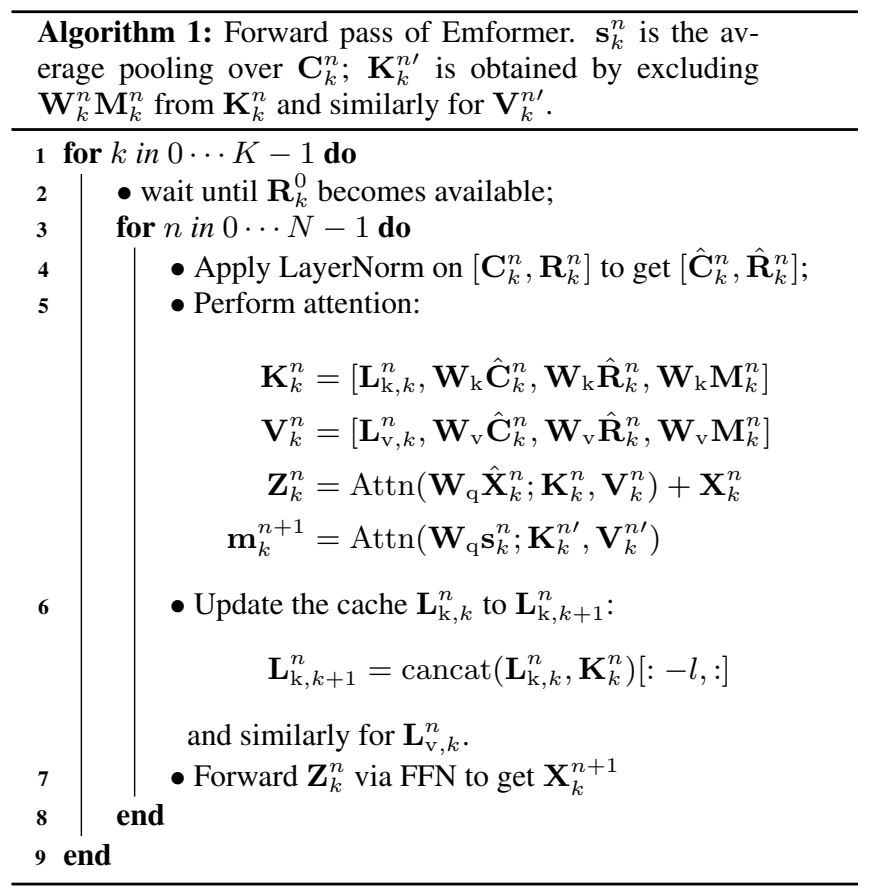

\section{Experiments}

This section compares the LSTM-based acoustic models with the transformer-based acoustic models for both low-latency and medium 
latency scenarios. Measuring user perceived latency is not straightforward and can be impacted by many aspects of the ASR systems. In this work, we define encoder induced latency (EIL) as the average difference between the time we received the physical signals and the time when the encoder emits the corresponding embeddings, assuming the actual computation is infinitely fast. In this way, we can focus on comparing the algorithmic latency introduced in different encoders. Under this definition, the EIL of models using block processing is calculated as the size of the right context plus half of the length of center segment. We compare word error rates (WERs) and real-time factors (RTFs) under the fixed EIL. RTFs are measured on a host with Intel Xeon D-2191A 18-core CPUs, while only 2 CPU threads are used for one utterance, one for forwarding through the acoustic model, and the other for search; 10 utterances are concurrently decoded when measuring RTFs.

All the experiments are using 80-dimensional log-Mel filter bank features at a $10 \mathrm{~ms}$ frame rate. Speed perturbation $[26 \mid$ and SpecAugment |27| without time warping are also used in all experiments. We compare encoders using different training criteria and/or modeling units. For hybrid systems, CTC criterion is used to train either a context-dependent grapheme (a.k.a. chenone) or a contextindependent wordpiece systems [28]. For neural transducer systems, wordpieces are used as the modeling unit. Kaldi [29| recipe is used to bootstrap the chenone system, while wordpiece systems do not need a bootstrap process. All the neural network models are trained using Adam optimizer [30]. Unless noted otherwise, all the models are trained by scanning the training data for 100 to 200 epochs. Learning rates increase to $1 \mathrm{e}-3$ in $20 \mathrm{~K}$ warming-up updates; then, it is fixed for 60 to 80 epochs; after that, the learning rate shrinks every epoch with factor 0.95 . In training deep transformer models, an auxiliary incremental loss [31] with weight 0.3 is used. All the models are trained using 32 Nvidia V100 GPUs with fp16 precision, except for the English and Spanish video ASR tasks, where 64 GPUs are used. Our training throughput ranges from $4 \mathrm{M}$ to $8 \mathrm{M}$ frames per second, which allows us to finish most of the experiments in less than 3 days. To get the best RTFs, all trained models are quantized to INT8, where each channel (row) in a matrix has its own quantizer. Compared with the fp16 model, per-channel quantization roughly costs us $1.2 \%$ relative WER loss but doubles the neural network evaluation speed. More details about our training and inference setup can be found in [11, 16].

\subsection{Comparison in Low latency Tasks}

To compare encoders in the low latency scenarios, we used our internal personal assistant dataset that contains queries like calling (e.g., "call Alex") and other types of voice queries (e.g., "what's the weather in Menlo Park?"). Our training set is made up of two manually-transcribed de-identified in-house corpora with no personally identifiable information (PII). The first corpus comprises $15.7 \mathrm{M}$ utterances ( $12.5 \mathrm{~K}$ hours) in the voice assistant domain recorded by $20 \mathrm{~K}$ crowd-sourced workers on mobile devices. The second corpus contains $1.2 \mathrm{M}$ voice commands ( $1 \mathrm{~K}$ hours) sampled from the production traffic of a smart speaker after the wakeword is activated. Utterances from this corpus are further morphed in an effort to deidentify the user. We distort each utterance in the first corpus twice using simulated Room Impulse Response (RIR) and additive background noise extracted from public videos. Utterances in the second corpus are already processed by a far-field frontend, so there is no need for RIR distortion. We create a few copies of each utterance in this corpus using speed perturbation and add additive background noise. The final distorted train data contains $56.1 \mathrm{M}$ utterances $(47 \mathrm{~K}$ hours). The evaluation data consists of $22.3 \mathrm{~K}$ hand-transcribed deidentified utterances from volunteer participants in the in-house pro- gram, which consists of employee households that have agreed to have their voice activity reviewed and tested. Every utterance in this set has an associated contact list, which we use for on-the-fly personalization for calling queries. These evaluation utterances are divided into two parts: name comprises $7 \mathrm{~K}$ utterances that have at least one entity name in the transcription, and the reset $15.3 \mathrm{~K}$ utterances form the general test set.

Uni-directional LSTM and Emformer-based hybrid systems are trained on this task using $8 \mathrm{~K}$ chenones as the modeling units. The current frame is stacked with 7 future frames as the input feature vector to the LSTM network. After the first layer, the input sequence is sub-sampled at a rate of $4: 1$. The LSTM network has 5 layers, each with 1200 cells, resulting in a model size of $65 \mathrm{M}$ parameters. To speed up inference, 10 frames $(100 \mathrm{~ms})$ are grouped as a batch for one forward through the LSTM network. Together with the 7-frame look-ahead in the frontend, this yields an EIL of 120ms. In Emformer, a linear layer first projects the 80-dimensional feature vectors to 128 dimensions, then every 4 such vectors are stacked and form a sequence of 512-dimensional feature vectors at a frame rate of 40ms. Two Emformer configurations which yield similar EILs as the baseline LSTM are used: in the first configuration, the center segment size is set as $120 \mathrm{~ms}$, and the right context is $80 \mathrm{~ms}$, which gives an EIL of 140ms; in the second configuration center segment size is $80 \mathrm{~ms}$, and the right context is $40 \mathrm{~ms}$, which gives an EIL of $80 \mathrm{~ms}$. Both configurations use a left context length of $800 \mathrm{~ms}$. Since the left context covers 7 to 10 past segments, there is no need to use memory in this scenario, thus we set the memory bank size to 0 . Both configurations use 18 layers of Emformer layers with 8 attention heads and 64 dimensions per head and 2048 as the FFN network dimension, yielding models with 60M parameters.

\begin{tabular}{|c|c|c|c|c|c|}
\hline $\begin{array}{l}\text { Train } \\
\text { (hrs) }\end{array}$ & Model & $\begin{array}{l}\text { EIL } \\
(\mathrm{ms})\end{array}$ & name & $\begin{array}{l}\text { st set } \\
\text { general }\end{array}$ & RTF \\
\hline \multirow{3}{*}{$4.7 \mathrm{~K}$} & LSTM & 120 & 10.89 & 5.43 & 0.194 \\
\hline & \multirow{2}{*}{ Emformer } & 140 & 8.05 & 3.94 & 0.227 \\
\hline & & 80 & 8.21 & 4.12 & 0.276 \\
\hline $47 \mathrm{~K}$ & Emformer & 80 & 6.83 & 4.05 & 0.275 \\
\hline
\end{tabular}

Table 1: WER/RTF comparison of LSTM with Emformer in a task requires low latency.

Results are presented in Table 1 In the first block, we first performed a quick turn-around experiment where only $10 \%$ of training data is used. With similar EILs (120ms vs 140ms), Emformer model significantly outperforms the LSTM baseline by $24-26 \%$ on both test sets, with slightly increased RTF. When further reducing the latency induced by encoder to $80 \mathrm{~ms}$, WERs are slightly increased. It also comes at the cost of RTFs (0.227 to 0.276) since the Emformer network needs to be evaluated more frequently on small batches. Lastly, by scaling up the training data to $47 \mathrm{~K}$ hours, another $16.8 \%$ WER reduction is observed on the name test set.

\subsection{Comparison in Medium Latency Tasks}

For medium latency scenarios, we compare the LCBLSTM-based encoder with Emformer on our internal video ASR task. 4 languages, en (English), es (Spanish), vi (Vietnamese) and de (German), are used in the comparison. The training data are extracted from videos shared publicly by users, where only the audio part is used. These data are de-identified: both transcribers and researchers do not have access to any user-identifiable information. For each language, besides a dev set for development purpose, there are 3 test sets in different conditions: clean, noisy and extreme. A voice activity detector (VAD) is used to segment whole audios into chunks which 
are not longer than 45 seconds ${ }^{2}$ Decoding is performed on these chunks. Training data are first aligned against reference hypothesis, and segmented into chunks with a maximum duration of 10 seconds. Training and test set sizes are summarized in Table 2 Furthermore, on $e n$, in additional to the $39.4 \mathrm{~K}$ hours supervised training data, we also prepared $2.16 \mathrm{M}$ hours unsupervised training data, whose transcription is obtained by sending de-identified, user-uploaded public videos to our internal automatic transcription service. No human effort is involved in transcribing these unsupervised data. In total, we have $2.2 \mathrm{M}$ hours semi-supervised data for the en task. To the best authors' knowledge, this is so far the largest speech recognition task ever published.

\begin{tabular}{|c||c|ccc|}
\hline language & Train & clean & noisy & extreme \\
\hline \hline$d e$ & $9.1 \mathrm{~K}$ & 24.9 & 24.7 & 42.3 \\
$v i$ & $10.2 \mathrm{~K}$ & 24.3 & 24.5 & 49.6 \\
$e s$ & $21.2 \mathrm{~K}$ & 26.7 & 26.3 & 49.7 \\
$e n$ & $39.4 \mathrm{~K}$ & 24.2 & 24.4 & 46.6 \\
\hline
\end{tabular}

Table 2: Training data and test sets sizes (in hours) for 4 languages.

Due to the differences in language characteristic, latency requirement, and size of training data, the architecture of ASR system for these four languages are slightly different:

- For vi sub task, chenone systems are trained using the CTC criterion. The LCBLSTM encoder consists of 5 layers, each with 800 hidden dimensions per direction. After the first and second layers, two 2:1 sub-samplings are performed, respectively. This yields a stride 4 encoder. Input to the Emformer network is first projected to 128-dimension, then 4 frames are stacked together to form an input sequence with a $40 \mathrm{~ms}$ frame rate. The Emformer encoder consists of 26 layers, each with 8 attention heads and 64 dimensions per head. During both training and inference, the maximum memory bank size is 4. For both LCBLSTM and Emformer, the center segment size is $1480 \mathrm{~ms}$, and the right context size is $320 \mathrm{~ms}$, i.e., EIL is $1060 \mathrm{~ms}$. The left context size in Emformer is $800 \mathrm{~ms}$.

- For de and es sub tasks, we trained neural transducer systems with 2047 wordpieces following setups in [32]. The LCBLSTM and Memformer encoders architecture follows the vi sub task, except the center segment size is $800 \mathrm{~ms}$, and the right context size is $320 \mathrm{~ms}$, which means EIL is $720 \mathrm{~ms}$. The left context size in Emformer is $800 \mathrm{~ms}$. In the predictor of the transducer, the wordpiece tokens are first represented by 256-dimensional embeddings before going through two LSTM layers, each with 512 hidden nodes, followed by a linear projection to 1024-dimension before the joiner; in the joiner, the combined embeddings from the encoder and the predictor first go through a Tanh activation and then another linear projection to 2048. For deeper models such as transformer and Emformer, both cross entropy loss and neural transducer loss are used to compute auxiliary loss, which is found to improve WERs [33].

- For en task, hybrid systems are trained using CTC criterion and wordpiece as the modeling unit. As demonstrated in [28], a large stride 8 in encoder can be used to speed up the inference without much impact on WER. Since en task has more training data, we increase the model capacity for both LCBLSTM and Emformer: LCBLSTM has 6 layers with each layer 1000 hidden cells; three 2:1 subsampling is performed after the first three layers respectively; Input to the Emformer network is first projected to 64-dimension, then 8 frames are stacked together to form an input sequence with an $80 \mathrm{~ms}$ frame rate. Emformer's depth is increased to 36 layers.

\footnotetext{
${ }^{2}$ Hybrid systems with LCBLSTM and Emformer can decode unlimited length of audio with only 2-3\% relatively WER increase.
}

The center segment size and right context size are kept the same as the one in $e s$ and de sub tasks. Furthermore, we also trained both LSTM-based and transformer-based acoustic models on the $2.2 \mathrm{M}$ hours semi-supervised data. Due to the sheer volume of training data, we only scan the training data by 3 epochs, and save the checkpoints for every $5 \mathrm{~K}$ steps. This allows us to finish training in 5 days. - For vi, es and en, the corresponding systems which use transformerbased encoders are also trained to show the upper bound of transformerbased acoustic models. 3 VGG blocks are used as not only a learnable frontend but also to add relative positional embedding information to the input of transformer layers. See [11] for more details. For vi and es, 24 transformer layers are used. For en, 36 transformer layers are used.

Results are presented in Table 3 Under the same latency constraint, except for extreme on the $v i$ sub task, Memformer consistently outperforms LCBLSTM, yielding $11.2 \%$ - 19.8\% WER reductions (WERR) on clean, $9.3 \%$ to $18.1 \%$ on noisy and $3.6 \%$ to $15.6 \%$ on extreme. Emformer also enjoys the fully parallelized computation within each forwarding step, which reduces the inference RTF over LCBLSTM by 2 to 3 times. On the other hand, compared with transformer-based model which does not have latency constraint, Emformer stills consistently lags behind by $3 \%$ to $11 \%$, which indicates there is still some space for accuracy improvement. Lastly, when training on the 2.2M-hour semi-supervised en data, both LCBLSTM and Emformer get over 10\% WER reduction on $e x$ treme test set over the supervised baselines; transformer gets even large gains, $7.4 \%$ on clean, $10 \%$ on noisy and $15 \%$ on extreme. Again the increased gap between transformer and Emformer indicates there are still some room of improvement for streamable transformers.

\begin{tabular}{|c|c|c|c|c|c|}
\hline Lang/System & Encoder & clean & noisy & extreme & RTF \\
\hline \multirow{2}{*}{$\begin{array}{c}d e \\
\text { transducer }\end{array}$} & LCBLSTM & 13.39 & 14.46 & 15.71 & 0.22 \\
\hline & Emformer & 10.74 & 11.84 & 13.26 & 0.11 \\
\hline \multirow{2}{*}{$v i$} & LCBLSTM & 11.34 & 16.12 & 40.96 & 0.19 \\
\hline & Emformer & 9.69 & 14.62 & 42.36 & 0.09 \\
\hline hybrid & $\operatorname{Trf}$ & 9.00 & 13.71 & 39.27 & 0.09 \\
\hline \multirow[b]{2}{*}{ es } & LCBLSTM & 10.24 & 13.58 & 23.23 & 0.22 \\
\hline & Emformer & 8.89 & 12.01 & 20.70 & 0.11 \\
\hline transducer & $\operatorname{Trf}$ & 8.62 & 11.63 & 19.78 & 0.11 \\
\hline & LCBLSTM & 11.90 & 16.06 & 22.49 & 0.27 \\
\hline & Emformer & 10.56 & 14.51 & 21.69 & 0.09 \\
\hline hybrid & $\operatorname{Trf}$ & 9.96 & 13.85 & 20.80 & 0.11 \\
\hline \multirow{3}{*}{$\begin{array}{c}e n \\
\text { hybrid } \\
(2.2 \mathrm{M} \text { hrs })\end{array}$} & LCBLSTM & 10.56 & 14.29 & 19.73 & 0.27 \\
\hline & Emformer & 10.54 & 13.98 & 19.51 & 0.09 \\
\hline & $\operatorname{Trf}$ & 9.22 & 12.47 & 17.68 & 0.09 \\
\hline
\end{tabular}

Table 3: Comparison of LCBLSTM with Emformer in medium latency scenarios. Transformer, which can be only used in offline scenarios, are also shown in the table for vi,es and en.

\section{Conclusions}

In this work, we compare the LSTM-based acoustic models with transformer-based ones for a range of large scale speech recognition tasks. Our results show that for low latency, voice assistant task, Emformer, a streamable transformer, gets $24 \%$ to $26 \%$ relative WERRs, compared with LSTM. For medium latency, video captioning task, compared with LCBLSTM, Emformer gets significant WERR across four languages and 2-3 times RTF reduction. Results on a task with $2.2 \mathrm{M}$ hours semi-supervised training data, indicate that there are still room for improvement for Emformer. Combining with convolution network to reduce model size and computational cost (e.g. [34]) can be our future work. 


\section{References}

[1] G. Hinton, L. Deng, D. Yu, et al., "Deep neural networks for acoustic modeling in speech recognition," IEEE Signal processing magazine, vol. 29, 2012.

[2] F. Seide, G. Li, and D. Yu, "Conversational speech transcription using context-dependent deep neural networks," in Proc. Interspeech, 2011.

[3] H. Sak, A. Senior, and F. Beaufays, "Long short-term memory recurrent neural network architectures for large scale acoustic modeling," in Proc. Interspeech, 2014.

[4] O. Abdel-Hamid, A. Mohamed, H. Jiang, et al., "Convolutional neural networks for speech recognition," IEEE/ACM Transactions on audio, speech, and language processing, vol. 22, no. 10, pp. 1533-1545, 2014.

[5] Y. Zhang, G. Chen, D. Yu, et al., "Highway long shortterm memory RNNs for distant speech recognition," in Proc. ICASSP. IEEE, 2016, pp. 5755-5759.

[6] V. Peddinti, D. Povey, and S. Khudanpur, "A time delay neural network architecture for efficient modeling of long temporal contexts," in Proc. Interspeech, 2015.

[7] S. Zhang, H. Jiang, S. Wei, and L. Dai, "Feedforward sequential memory neural networks without recurrent feedback," arXiv preprint arXiv:1510.02693, 2015.

[8] A. Vaswani, N. Shazeer, N. Parmar, et al., "Attention is all you need," in Proc. NIPS, 2017, pp. 5998-6008.

[9] J. Devlin, M.-W. Chang, K. Lee, et al., "Bert: Pre-training of deep bidirectional transformers for language understanding," arXiv preprint arXiv:1810.04805, 2018.

[10] S. Karita, N. Chen, T. Hayashi, et al., "A Comparative Study on Transformer vs RNN in Speech Applications," arXiv preprint arXiv:1909.06317, 2019.

[11] Y. Wang, A. Mohamed, D. Le, C. Liu, A. Xiao, et al., "Transformer-based acoustic modeling for hybrid speech recognition," in Proc. ICASSP, 2020, pp. 6874-6878.

[12] A. Gulati, J. Qin, C.-C. Chiu, N. Parmar, et al., "Conformer: Convolution-augmented Transformer for Speech Recognition," arXiv preprint arXiv:2005.08100, 2020.

[13] Q. Zhang, H. Lu, H. Sak, A. Tripathi, E. McDermott, S. Koo, and S. Kumar, "Transformer transducer: A streamable speech recognition model with transformer encoders and RNN-T loss," in Proc. ICASSP, 2020, pp. 7829-7833.

[14] N. Moritz, T. Hori, and J. L. Roux, "Streaming automatic speech recognition with the transformer model," arXiv preprint arXiv:2001.02674, 2020.

[15] C. Wu, Y. Wang, Y. Shi, C.-F. Yeh, and F. Zhang, "Streaming Transformer-based Acoustic Models Using Self-attention with Augmented Memory," arXiv preprint arXiv:2005.08042, 2020.

[16] Y. Shi, Y. Wang, C. Wu, C.-C. Yeh, et al., "Emformer: efficient low latency streaming transformer acoustic model for speech recognition," arXiv preprint arXiv:2010.10759.

[17] Alex Graves, "Sequence transduction with recurrent neural networks," arXiv preprint arXiv:1211.3711, 2012.

[18] H. A. Bourlard and N. Morgan, Connectionist speech recognition: a hybrid approach, vol. 247, Springer Science \& Business Media.
[19] M.-Y. Hwang and X. Huang, "Subphonetic modeling with markov states-senone," in Proc. ICASSP, 1992, vol. 1, pp. 3336.

[20] D. Le, X. Zhang, W. Zheng, et al., "From Senones to Chenones: Tied Context-Dependent Graphemes for Hybrid Speech Recognition," arXiv preprint arXiv:1910.01493, 2019.

[21] A. Graves, S. Fernández, F. Gomez, and J. Schmidhuber, "Connectionist temporal classification: labelling unsegmented sequence data with recurrent neural networks," in Proceedings of the 23rd international conference on Machine learning, 2006, pp. 369-376.

[22] B. Kingsbury, "Lattice-based optimization of sequence classification criteria for neural-network acoustic modeling," in Proc. ICASSP, 2009, pp. 3761-3764.

[23] M. Schuster and K. Nakajima, "Japanese and korean voice search,” in Proc. ICASSP, 2012, pp. 5149-5152.

[24] J. Lei Ba, J. Kiros, and G. E. Hinton, "Layer normalization," arXiv preprint arXiv:1607.06450, 2016.

[25] D. Bahdanau, K. Cho, and Y. Bengio, "Neural machine translation by jointly learning to align and translate," arXiv preprint arXiv:1409.0473, 2014.

[26] T. Ko, V. Peddinti, D. Povey, et al., "Audio augmentation for speech recognition," in Proc. Interspeech, 2015.

[27] D. S. Park, W. Chan, Y. Zhang, et al., "Specaugment: A simple data augmentation method for automatic speech recognition," arXiv preprint arXiv:1904.08779, 2019.

[28] "faster, simpler and more accurate hybrid asr systems using wordpieces," .

[29] D. Povey, A. Ghoshal, G. Boulianne, et al., "The kaldi speech recognition toolkit," in IEEE Workshop on Automatic Speech Recognition and Understanding, 2011.

[30] D. P. Kingma and J. Ba, "Adam: A method for stochastic optimization," arXiv preprint arXiv:1412.6980, 2014.

[31] A. Tjandra, C. Liu, F. Zhang, et al., "Deja-vu: Double Feature Presentation and Iterated loss in Deep Transformer Networks," To appear ICASSP, 2020.

[32] X. Zhang, F. Zhang, C. Liu, K. Schubert, J. Chan, et al., "Benchmarking LF-MMI, CTC and RNN-T criteria for streaming ASR," submitted to IEEE Spoken Language Technology workshop, 2021.

[33] C. Liu, F. Zhang, D. Le, S. Kim, et al., "Improving RNN transducer based ASR with auxiliary tasks," submitted to IEEE Spoken Language Technology workshop, 2021.

[34] C.-F. Yeh, Y. Wang, S. Yang, C. Wu, F. Zhang, et al., "Streaming Attention-Based Models with Augmented Memory for End-to-End Speech Recognition," submitted to IEEE Spoken Language Technology workshop, 2021. 